\title{
Subcortical Representation of Non-Fourier Image Features
}

\author{
Ari Rosenberg, ${ }^{1}$ T. Robert Husson, ${ }^{1}$ and Naoum P. Issa ${ }^{2}$ \\ ${ }^{1}$ Committee on Computational Neuroscience and ${ }^{2}$ Department of Neurobiology, University of Chicago, Chicago, Illinois 60637
}

\begin{abstract}
A fundamental goal of visual neuroscience is to identify the neural pathways representing different image features. It is widely argued that the early stages of these pathways represent linear features of the visual scene and that the nonlinearities necessary to represent complex visual patterns are introduced later in cortex. We tested this by comparing the responses of subcortical and cortical neurons to interference patterns constructed by summing sinusoidal gratings. Although a linear mechanism can detect the component gratings, a nonlinear mechanism is required to detect an interference pattern resulting from their sum. Consistent with in vitro retinal ganglion cell recordings, we found that interference patterns are represented subcortically by cat LGN Y-cells, but not X-cells. Linear and nonlinear tuning properties of LGN Y-cells were then characterized and compared quantitatively with those of cortical area 18 neurons responsive to interference patterns. This comparison revealed a high degree of similarity between the two neural populations, including the following: (1) the representation of similar spatial frequencies in both their linear and nonlinear responses, (2) comparable orientation selectivity for the high spatial frequency carrier of interference patterns, and (3) the same difference in their temporal frequency selectivity for drifting gratings versus the envelope of interference patterns. The present findings demonstrate that the nonlinear subcortical Y-cell pathway represents complex visual patterns and likely underlies cortical responses to interference patterns. We suggest that linear and nonlinear mechanisms important for encoding visual scenes emerge in parallel through distinct pathways originating at the retina.
\end{abstract}

\section{Introduction}

To extract perceptually salient image features, the visual system relies on both linear and nonlinear mechanisms. Linear mechanisms detect spatiotemporal variation in mean luminance (Fourier image features, such as luminance defined sinusoidal gratings) (Watson and Ahumada, 1983; Adelson and Bergen, 1985; Baker and Issa, 2005; Mante and Carandini, 2005). Nonlinear mechanisms detect features defined by the higher-order statistics of the luminance profile, including spatiotemporal variation in contrast or texture (non-Fourier image features, such as interference patterns) (Chubb and Sperling, 1988; Fleet and Langley, 1994; Lu and Sperling, 2001). Non-Fourier features play an important role in many visual tasks including boundary detection (Rivest and Cavanagh, 1996; Leventhal et al., 1998; Song and Baker, 2007), velocity computation (Chubb and Sperling, 1988; Albright, 1992), depth analysis (Langley et al., 1998; Hegdé et al., 2004; Tanaka and Ohzawa, 2006), and the analysis of form (Sáry et al., 1993; Bellefeuille and Faubert, 1998).

Although the importance of non-Fourier processing is well recognized, it is debated whether it begins subcortically or in cortex. Non-Fourier motion is perceived by some animals lacking cortical circuitry, for example fish larvae (Orger et al., 2000) and flies (Theobald et al., 2008), and has been shown to elicit

Received July 8, 2009; revised Nov. 3, 2009; accepted Nov. 29, 2009.

This work was supported by Department of Homeland Security Fellowship DE-AC05-000R22750 (A.R.) and grants from the Brain Research Foundation and Mallinckrodt Foundation (N.P.I.). We thank Atul K. Mallik and Lisa Wang for assistance during experimental sessions, Curtis L. Baker Jr for providing us with circular variance measurements of carrier orientation selectivity in area 18, and David J. Freedman and Steven K. Shevell for helpful comments and suggestions on this manuscript.

Correspondence should be addressed to Naoum P. Issa at naoumpissa@gmail.com.

DOI:10.1523/JNEUROSCI.3258-09.2010

Copyright $\odot 2010$ the authors $\quad 0270-6474 / 10 / 301985-09 \$ 15.00 / 0$ responses from guinea pig retinal ganglion Y-cells in vitro (Demb et al., 2001). However, the suggestion of a subcortical locus has not been widely accepted because the representation of nonFourier features within the mammalian visual system appears to be incompatible with a retinal source. Cortical electrophysiology in cats (Mareschal and Baker, 1998b) and monkeys (von der Heydt and Peterhans, 1989) as well as human psychophysics (Langley et al., 1996; Dakin and Mareschal, 2000) have suggested that the neural representation of non-Fourier features is constructed from an earlier cortex-like representation of the visual scene. Within the mammalian visual system, the prevailing model for the representation of non-Fourier features is thus a hierarchical one: the visual scene is represented in a dominantly linear manner through primary visual cortex after which nonFourier features are extracted via a nonlinear intracortical transformation (Baker and Mareschal, 2001).

To examine whether cortical responses to non-Fourier features may derive from an earlier subcortical representation, we compared how neurons in the lateral geniculate nucleus (LGN) and area 18 of the cat respond to non-Fourier features. Using single-unit extracellular electrophysiology, we recorded the activity of LGN X- and Y-cells during the presentation of interference patterns. We found that whereas none of the $\mathrm{X}$-cells responded to interference patterns, all of the Y-cells responded robustly. We then compared Y-cell spatiotemporal tuning properties with those of area 18 neurons and found a high degree of similarity in how the two neural populations responded to non-Fourier features. In conjunction with known Y-cell cortical projection patterns (Stone and Dreher, 1973; Humphrey et al., 1985), these results support the hypothesis that the cortical representation of non-Fourier features originates subcortically with the Y-cell pathway. We suggest that the neural representations of Fourier 
and non-Fourier features emerge in parallel, not hierarchically, through the canonical $\mathrm{X}$ - and $\mathrm{Y}$-cell pathways originating at the retina.

\section{Materials and Methods}

Animal preparation. All procedures were approved by the University of Chicago Institutional Animal Care and Use Committee. Anesthesia was induced using a mixture of ketamine $(15 \mathrm{mg} / \mathrm{kg})$ and acepromazine $(0.05$ $\mathrm{mg} / \mathrm{kg}$ ) given intramuscularly and supplemented with isoflurane. Anesthesia was maintained with isoflurane $1-2 \%$ except in one animal that was maintained using thiopental (loading dose $20-30 \mathrm{mg} / \mathrm{kg}$, i.v., maintenance $2-3 \mathrm{mg} / \mathrm{kg}$ doses, i.v., as needed). Animals received Baytril subcutaneously $(2.5-5 \mathrm{mg} / \mathrm{kg}$, s.c.) as prophylaxis against infection, dexamethasone $(1.0-2.0 \mathrm{mg} / \mathrm{kg}$, s.c.) to reduce cerebral edema, and atropine $(0.04 \mathrm{mg} / \mathrm{kg}$, s.c.) to decrease tracheal secretions. Ophthalmic atropine $(1 \%)$ and phenylephrine $(10 \%)$ were instilled in the eyes to dilate the pupils and retract the nictitating membrane, respectively. Lactated Ringer's solution (LRS) with $2.5 \%$ dextrose was given through a venous cannula $(7-10 \mathrm{ml} / \mathrm{kg} / \mathrm{h})$. Pancuronium bromide was delivered in the LRS solution $(0.1 \mathrm{mg} / \mathrm{kg}$ i.v. induction, 0.125 $\mathrm{mg} / \mathrm{kg} / \mathrm{h}$ continuous) to maintain paralysis. Core temperature was maintained around $38^{\circ} \mathrm{C}$ with a water heating blanket (Gaymar Industries). Positive pressure ventilation $\left(1: 2 \mathrm{O}_{2}: \mathrm{N}_{2} \mathrm{O}\right)$ was adjusted to maintain end-tidal $\mathrm{CO}_{2}$ between $3.8 \%$ and $4.3 \%$ with a peak inspiratory pressure of $13-21 \mathrm{~cm} \mathrm{H}_{2} \mathrm{O}$. ECG and EEG were monitored throughout the experiment. Contact lenses were used to focus the eyes on the monitor at a distance of $40 \mathrm{~cm}$. In LGN experiments, the focus was confirmed by identifying the lens power that maximized the high spatial frequency (SF) cutoff of X-cells.

Electrophysiology. Extracellular action potentials were recorded in the A and C layers of the LGN and area 18 of adult cats using epoxy-coated, high-impedance (1-10 M $\Omega$ ) tungsten electrodes (FHC). To reach the LGN, electrodes were lowered dorsoventrally through a craniotomy (Horsley-Clarke coordinates $\sim 9 \mathrm{~mm}$ lateral and $\sim 6 \mathrm{~mm}$ anterior). The LGN was identified by its stereotyped layer structure and the physiological responses of individual neurons. Neurons were classified as either Xor Y-type using standard methods based on a comparison of responses to high SF drifting and contrast-reversing gratings (Hochstein and Shapley, 1976a). An example of Y-cell responses are shown in supplemental Figure 1 (available at www.jneurosci.org as supplemental material). X-cells were recorded in the A-layers only $(n=27)$. Y-cells were recorded in the A-layers $(n=40)$ and within the first $635 \mu \mathrm{m}$ of the C-layers $(n=14)$. Some of the cells recorded in the C-layers had markedly lower spatiotemporal frequency preferences than those recorded in the A-layers, suggesting that a few nonlinear W-cells may have been included in these analyses (Sur and Sherman, 1982). However, no qualitative differences between the response properties of cells recorded in the A- and C-layers were apparent. The receptive field positions of $24 \mathrm{Y}$-cells were measured. Eccentricity ranged between $4.4^{\circ}$ and $25.8^{\circ}$ with an average of $14.2^{\circ} \pm 5.4^{\circ}$ $\mathrm{SD}$. Area 18 was targeted following optical imaging, using the area $17-18$ border defined functionally as a shift from high to low SF preference running mediolaterally in the anterior-posterior direction. Action potentials were amplified and filtered at $5 \mathrm{kHz}$ (A-M Systems, Model 1800), then digitally sampled at $20 \mathrm{kHz}$ and stored for off-line spike sorting (CED, Micro 1400).

Stimuli. Visual stimuli were generated by computer and displayed monocularly on a gamma-corrected 21 inch cathode ray tube monitor with a mean luminance $26 \mathrm{~cd} / \mathrm{m}^{2}$ using the Psychophysics Toolbox extensions (Brainard, 1997) for MATLAB (The MathWorks). The linear relationship between stimulus intensity commanded by the software and the output luminance of the monitor was confirmed in two ways: (1) with a light meter (Konica Minolta, Model LS-100) and (2) as previously described (Zhang et al., 2007), by performing a fast Fourier transform on visual stimuli photographed with a Dalsa 1 M30 CCD camera. All stimuli were presented as full-field images covering $43^{\circ} \times 57^{\circ}$ of visual space, and the monitor was refreshed at $100 \mathrm{~Hz}$. Stimuli consisted of high contrast ( $80 \%$ Michelson) drifting and contrast-reversing sinusoidal gratings as well as three-component interference patterns (Fig. 1, Eq. 1). In this equation, $\omega_{\mathrm{C}}$ is the vector defining the carrier spatiotemporal frequencies, $\omega_{\mathrm{E}}$ is the vector defining the envelope frequencies, and $\chi$ is the vector defining the space and time dimensions $(x, y, t)$. Contrast-reversing gratings are by definition two-component interference patterns that have a temporal envelope but no spatial envelope. We reserve the term "interference pattern" to refer strictly to three-component interference patterns of the form described by Equation 1, which have both spatial and temporal envelopes (Fig. 1C), as follows:

$$
\begin{aligned}
I(x, y, t)=\cos \left(\omega_{\mathrm{C}} \cdot \chi\right)+0.5 \cdot\left\{\cos \left(\left[\omega_{\mathrm{C}}-\omega_{\mathrm{E}}\right] \cdot \chi\right)\right. \\
\left.+\cos \left(\left[\omega_{\mathrm{C}}+\omega_{\mathrm{E}}\right] \cdot \chi\right)\right\} .
\end{aligned}
$$

As in cortical studies of non-Fourier features, the envelope orientation and SF of the interference patterns presented to a cell were fixed near values similar to its linear preferences (measured using drifting sinusoidal gratings).X-cells were typically tested with nine interference patterns, using three carrier spatial frequencies and three carrier orientations, but sometimes more. For a Y-cell, the carrier SF was set near the preferred SF of the cell's nonlinear responses measured using contrast-reversing gratings. Interference patterns would not elicit $\mathrm{Y}$-cell responses if the carrier SF was too high to elicit a contrast-reversing response (supplemental Fig. 2 , available at www.jneurosci.org as supplemental material). Some Y-cells only responded to interference patterns if the carrier drifted, but the dependency of responses on carrier temporal frequency (TF) will be addressed in a future report. In analyses of carrier orientation and envelope TF tuning, the carrier TF was always zero to allow for a direct comparison between this dataset and those of previous studies conducted in cat area 18 (Mareschal and Baker, 1998a; Song and Baker, 2007). Each stimulus was presented between 4 and 12 times. Stimuli were presented statically for $250 \mathrm{~ms}$ before moving for a period lasting either 2 or $3 \mathrm{~s}$. A mean-level gray screen was presented for $500 \mathrm{~ms}$ between trials. Average firing rates were calculated over the drift duration.

Data analysis. The SF tuning of X-cells and the linear responses of Y-cells was assessed using drifting sinusoidal gratings. The preferred SF was estimated from a log-Gaussian fit to the measured SF tuning curve if the strongest response was not at the lowest tested SF, and was otherwise taken to be the lowest tested SF. For these fits, the median $r^{2}=0.92$ for $\mathrm{X}$-cells $(n=21)$ and $r^{2}=0.95$ for Y-cells $(n=39)$. The SF tuning of the nonlinear responses of Y-cells was assessed using contrast-reversing gratings. A log-Gaussian would not always fit these tuning curves (e.g., supplemental Fig. 3C,I, available at www.jneurosci.org as supplemental material), so the preferred SF was estimated by taking a weighted average. For a given SF, the corresponding weight was the amplitude of the neural response oscillating at twice the stimulus TF. A cell's preferred TF was estimated from a gamma function fit to the measured TF tuning curve. For these fits, the median $r^{2}=0.96$ for drifting sinusoidal gratings ( $n=$ $36), r^{2}=0.9$ for the envelope of interference patterns $(n=30)$, and $r^{2}=$ 0.98 for contrast-reversing gratings $(n=34)$. Summary data of cortical TF tuning curves were extracted from Mareschal and Baker (1998a) using the graph-tracing program DataThief. A cell's preferred orientation was estimated by calculating the vector average of its orientation tuning curve. Orientation selectivity was quantified as $V=1-$ circular variance $(\mathrm{CV})$, Equation 2, after baseline subtracting the data. The response at orientation $\theta_{i}$ is given by $R_{i}$. A value of $V=1$ indicates that the neuron responded to only one orientation, and a value of $V=0$ indicates that the neuron responded equally well to all orientations, as follows:

$$
V=\left|\frac{\sum_{i} R_{i} \exp \left(i 2 \theta_{i}\right)}{\sum_{i} R_{i}}\right| .
$$

The ability to discriminate the presentation of an interference pattern from a blank screen using the single-trial firing rates of individual neurons was quantified with the Gini coefficient $(G)$ from receiver operating characteristics (ROC) analysis (Fawcett, 2006). The Gini coefficient is calculated as twice the area under the ROC curve (AUC) minus 1 (Eq. 3). A value of $G=1$ indicates perfect discrimination, a value of $G=0$ indicates chance performance, and a negative value indicates that the interference pattern suppressed activity relative to 


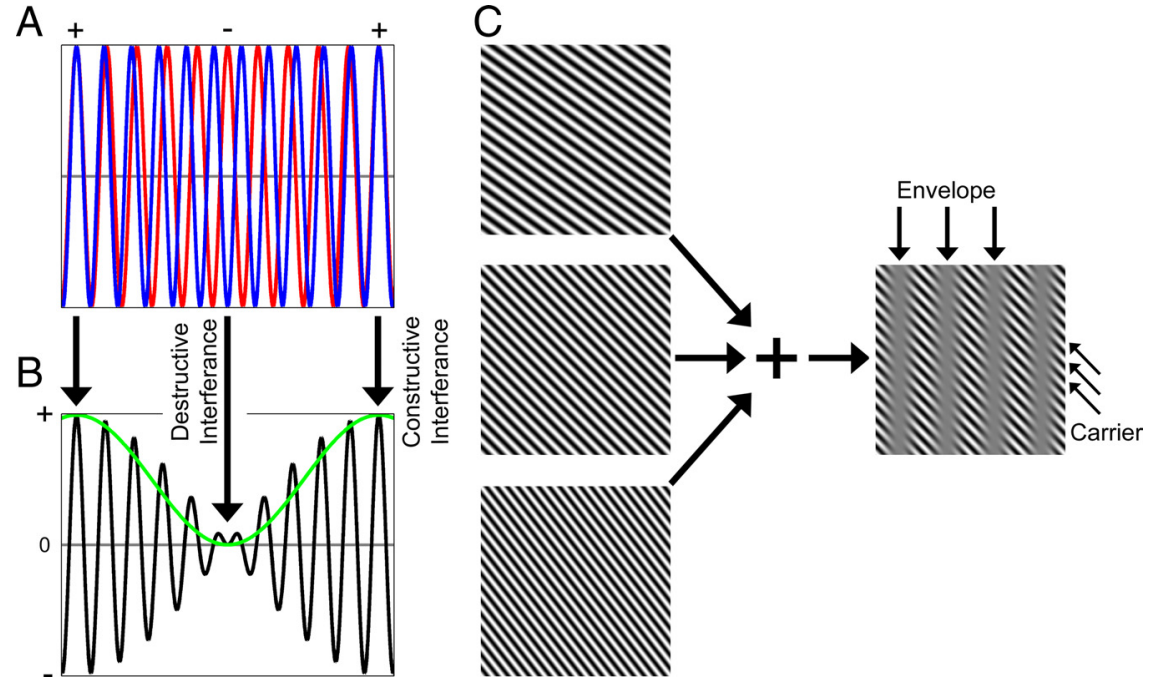

Figure 1. Constructing an interference pattern. $A$, Two sinusoids with slightly different frequencies are plotted in blue and red. They are initially in phase (left side, point indicated by a " + "), but the difference in their frequencies causes them to diverge until they are $180^{\circ}$ out of phase (middle of plot, point indicated by a " - "). They then reconverge into phase (right side, point indicated by a " $+")$. $\boldsymbol{B}$, The sum of the two sinusoids in $\boldsymbol{A}$ is plotted in black. Constructive and destructive interference results in an interference pattern or amplitude-modulated (AM) signal whose "envelope" (in green) oscillates at the difference frequency of the two sinusoids. C, A two-dimensional interference pattern, also called a contrast-modulated grating, is shown furthest to the right. The pattern is the sum of the three obliquely oriented high SF gratings to its left, but it appears vertically oriented with a low SF. (The component gratings are shown with the same contrast, but to construct the interference pattern, the components on the top and bottom had half the contrast of the one in the middle.) An interference pattern is succinctly described as a high SF carrier (here, obliquely oriented at $135^{\circ}$ ) whose contrast is modulated by a low SF envelope (here, vertically oriented). nonlinear responses that have been well studied in cortex (Mareschal and Baker, 1998a,b, 1999; Song and Baker, 2007). (The type of interference pattern used in this study is constructed by summing three high SF gratings, but is conveniently described as a high SF carrier whose contrast is modulated by a low SF envelope (Fig. 1). Although no low spatial frequencies are actually present in the stimulus, it elicits the perception of a pattern with the low SF and orientation of the envelope.) The responses of cat LGN and area 18 neurons to interference pattern were then compared quantitatively. Previous work has shown that $\sim 63 \%$ of neurons in area 18 respond to interference patterns (Zhou and Baker, 1994). Cat area 18 receives LGN input from Y-cells but not X-cells (Stone and Dreher, 1973; Humphrey et al., 1985) and also receives cortical input from area 17 (Price et al., 1994). As such, area 18 responses to interference patterns may reflect feedforward processing of the output of LGN Y-cells or area 17. Here we examine whether LGN Y-cell response properties can account for area 18 responses to interference patterns.
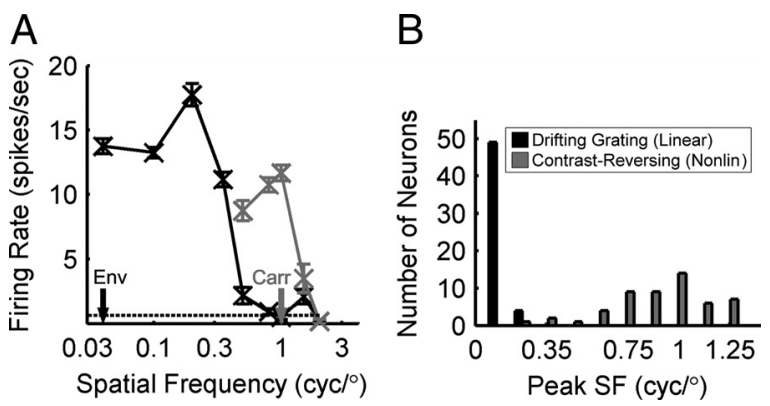

Figure 2. Spatial frequency tuning of $Y$-cell linear and nonlinear responses. $A$, SF tuning of an LGN Y-cell. Responses to drifting sinusoidal gratings are used to define a linear SF tuning curve (solid black curve). Responses to contrast-reversing gratings are used to define a nonlinear SF tuning curve (solid gray curve). Also see supplemental Figure 1 (available at www.jneurosci.org as supplemental material). Baseline firing rate is indicated by the dotted black line. Error bars show SEM. Arrows mark the envelope and carrier spatial frequencies of the interference patterns used to study the neuron's non-Fourier responses. Note that at high spatial frequencies, the neuron responded to contrastreversing gratings but not drifting gratings. $\boldsymbol{B}$, Histogram of the preferred linear and nonlinear spatial frequencies of $\mathrm{Y}$-cells.

the blank screen response. For each cell, the maximally measured $G$ is reported as follows:

$$
G=2 \cdot \mathrm{AUG}-1
$$

\section{Results}

We characterized linear and nonlinear response properties of single neurons in the LGN using drifting sinusoidal gratings, contrast-reversing gratings, and interference patterns. Drifting sinusoidal gratings were used to study linear response properties, contrast-reversing gratings were used to classify cells as X- or Y-type and to perform an initial characterization of Y-cell nonlinear responses, and interference patterns were used to study

\section{The identification of $\mathrm{X}$ - and $\mathrm{Y}$-cells and measurements of} spatial frequency tuning

The characterization of an isolated LGN neuron began by identifying it as either an X- or a Y-cell based on its responses to drifting and contrast-reversing gratings. Both cell types respond to drifting gratings with modulations in firing rate that oscillate at the stimulus TF. These responses define a cell's "linear" SF tuning curve. Because X-cells exhibit predominantly linear spatial summation, their responses to drifting gratings accurately predict their responses to other stimuli including contrast-reversing gratings. Y-cells, however, exhibit nonlinear spatial summation and in response to high SF contrast-reversing gratings that elicit little or no linear response, their firing rate modulates at twice the stimulus TF (supplemental Fig. 1, available at www.jneurosci.org as supplemental material) (Hochstein and Shapley, 1976a,b; Victor, 1988). These responses define a Y-cell's "nonlinear" SF tuning curve. A neuron was thus classified as a Y-cell if its responses to high SF contrast-reversing gratings were "frequency-doubled" and otherwise classified as an X-cell.

The X-cells that were studied tended to prefer higher spatial frequencies than Y-cells, but preferences varied greatly (average $\mathrm{X}$-cell SF peak $=0.24 \mathrm{cyc}^{\circ} \pm 0.23 \mathrm{SD}, n=27$ ). In general, only $\mathrm{X}$-cells with SF tuning curves that peaked at low to moderate values were studied to ensure that the presented interference patterns only contained spatial frequencies within the cat visual acuity limit. The linear responses of Y-cells always preferred low spatial frequencies (average $\mathrm{SF}_{\text {peak }}=0.09 \mathrm{cyc} /{ }^{\circ} \pm 0.05 \mathrm{SD}, n=$ 54 ), whereas the nonlinear responses (measured using contrastreversing gratings) preferred high spatial frequencies (average $\mathrm{SF}_{\text {peak }}=0.91 \mathrm{cyc}^{\circ} \pm 0.23 \mathrm{SD}, n=54$ ) (Fig. 2). Y-cells in the A-layers tended to prefer slightly higher spatial frequencies for drifting gratings (average $\mathrm{SF}_{\text {peak }}=0.1 \mathrm{cyc}^{\circ} \pm 0.05 \mathrm{SD}, n=40$ ) than Y-cells in the C-layers (average $\mathrm{SF}_{\text {peak }}=0.07 \mathrm{cyc} /^{\circ} \pm 0.06$ $\mathrm{SD}, n=14$ ). A more pronounced difference between the layers 
was found for the preferred nonlinear spatial frequencies. The preferred nonlinear SF of A-layer Y-cells was on average $0.96 \mathrm{cyc}^{\circ} \pm 0.19 \mathrm{SD}(n=40)$ and for C-layer Y-cells it was $0.75 \mathrm{cyc} /{ }^{\circ} \pm 0.29 \mathrm{SD}$ $(n=14)$. The lower mean and larger SD found in the C-layers may indicate the inclusion of both Y- and nonlinear W-type cells (Sur and Sherman, 1982).

The observed SF preferences of the Y-cell linear and nonlinear responses were similar to those of cortical neurons. In area 18 , a previous study reported an average preferred linear SF of $0.08 \mathrm{cyc} /{ }^{\circ}$ and an average preferred high SF carrier for interference patterns of $1.09 \mathrm{cyc} /{ }^{\circ}$. The relative relationship between the preferred linear and nonlinear spatial frequencies of neurons in the LGN and cortex was similar. In the LGN, the preferred SF of a Y-cell's nonlinear response was on average $\sim 13$ times greater than its preferred linear SF. In area 18, a previous report found that a neuron's preferred carrier SF is on average $\sim 10$ times greater than its preferred linear SF (Mareschal and Baker, 1999).

\section{Subcortical responses to non-Fourier image features}

We used interference patterns to study non-Fourier responses in the LGN. As in cortical studies, the envelope SF was fixed at or slightly below the preferred SF of the linear responses and the carrier SF was chosen to be high enough that the individual component gratings would not elicit linear responses (representative parameter selections are marked in Figs. $2 A, 3 E$, and supplemental Fig. 3, available at www.jneurosci.org as supplemental material). For Y-cells, the carrier SF was selected to be near the preferred SF of the nonlinear responses measured using contrastreversing gratings. The responses of an $\mathrm{X}$ - and a Y-cell to drifting gratings and interference patterns are presented in Figure 3. The responses of the cells were qualitatively the same for drifting gratings, modulating at the stimulus TF when the SF was within the cell's linear passband and unmodulated when the SF was above that passband (Fig. $3 A, B, D, E$ ). However, the presentation of interference patterns whose components did not drive linear responses allowed the cells to be differentiated qualitatively. Whereas the Y-cell responded robustly at the envelope TF (Fig. $3 C)$, the interference pattern did not elicit a response from the $\mathrm{X}$-cell (Fig. 3F).

Across the population of X- and Y-cells, the ability to discriminate an interference pattern from a blank screen, a task that is impossible for a linear system, was quantified using the Gini coefficient $(G)$ from ROC analysis (Fig. 4) (Fawcett, 2006). A value of $G=0$ indicates chance performance and $G=1$ indicates perfect discrimination. For X-cells, the average $G=0.16 \pm 0.03$ $\operatorname{SEM}(n=27)$. The strongest X-cell interference pattern response in our sample $(G=0.46)$ is presented in Figure 3F. In six 2-s-long presentations, the cell fired 393 action potentials in response to a drifting grating at its preferred SF but fired only 8 action potentials during the presentation of an interference pattern. All of the Y-cells in our sample had a higher Gini coefficient than any of the X-cells. For Y-cells, the average $G=0.97 \pm 0.01$ SEM $(n=51)$,
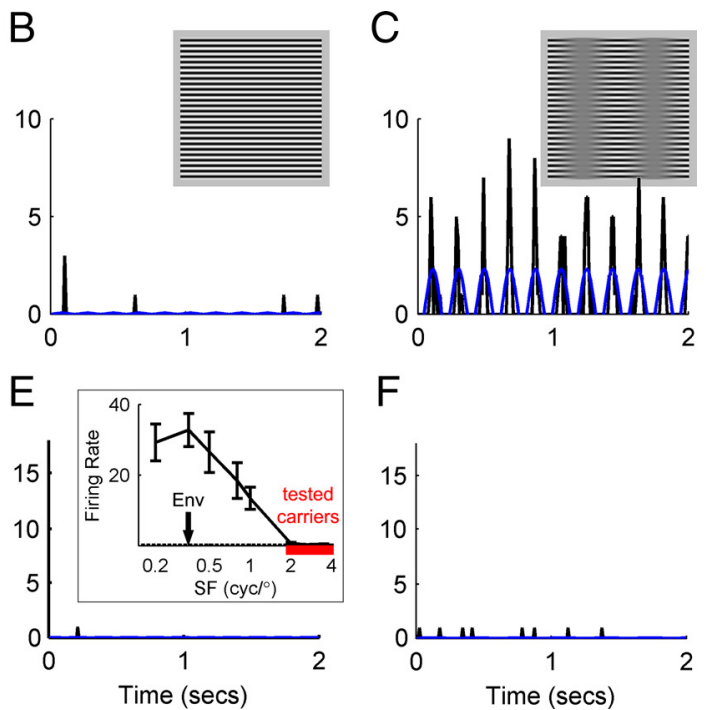

Figure 3. LGN responses to drifting gratings and interference patterns. Black traces show spike count histograms and blue traces show sinusoidal fits at the stimulus TF (the sinusoids are clipped at zero). $\boldsymbol{A}-\boldsymbol{C}$, Responses of the $Y$-cell whose SF tuning is

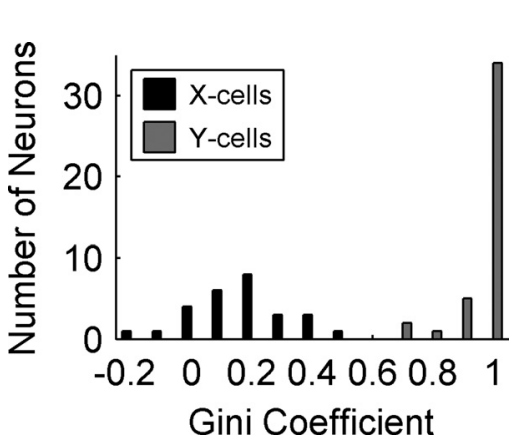

Figure 4. ROC analysis of $X$ - and $Y$-cell responses to interference patterns. The ability to discriminate an interference pattern from a blank screen using single-trial firing rates was quantified with the Gini coefficient. One indicates perfect discrimination, zero indicates chance performance, and negative values indicate that the cell tended to fire more action potentials during the presentation of a blank screen than an interference pattern. Note that the X-and Y-cell Gini coefficient distributions were non-overlapping and that the responses of the majority of $Y$-cells could be used to perfectly discriminate an interference pattern from a blank screen $(G=1)$.

indicating that Y-cells respond well to interference patterns. This result confirms an earlier report that Y-cells respond to nonFourier motion (Demb et al., 2001) and demonstrates that X-cells do not signal non-Fourier image features. Note that the lack of response from $\mathrm{X}$-cells also serves as a control demonstrating that interference pattern responses were not due to stimulus artifacts (see supplemental material, available at www.jneurosci.org).

\section{Orientation selectivity of the linear and nonlinear responses of LGN Y-cells}

Both psychophysical (Langley et al., 1996; Dakin and Mareschal, 2000) and cortical electrophysiology studies (Mareschal and Baker, 1998b; Song and Baker, 2007) have demonstrated that sensitivity to interference patterns is dependent on carrier orientation. To account for this, the representation of non-Fourier 

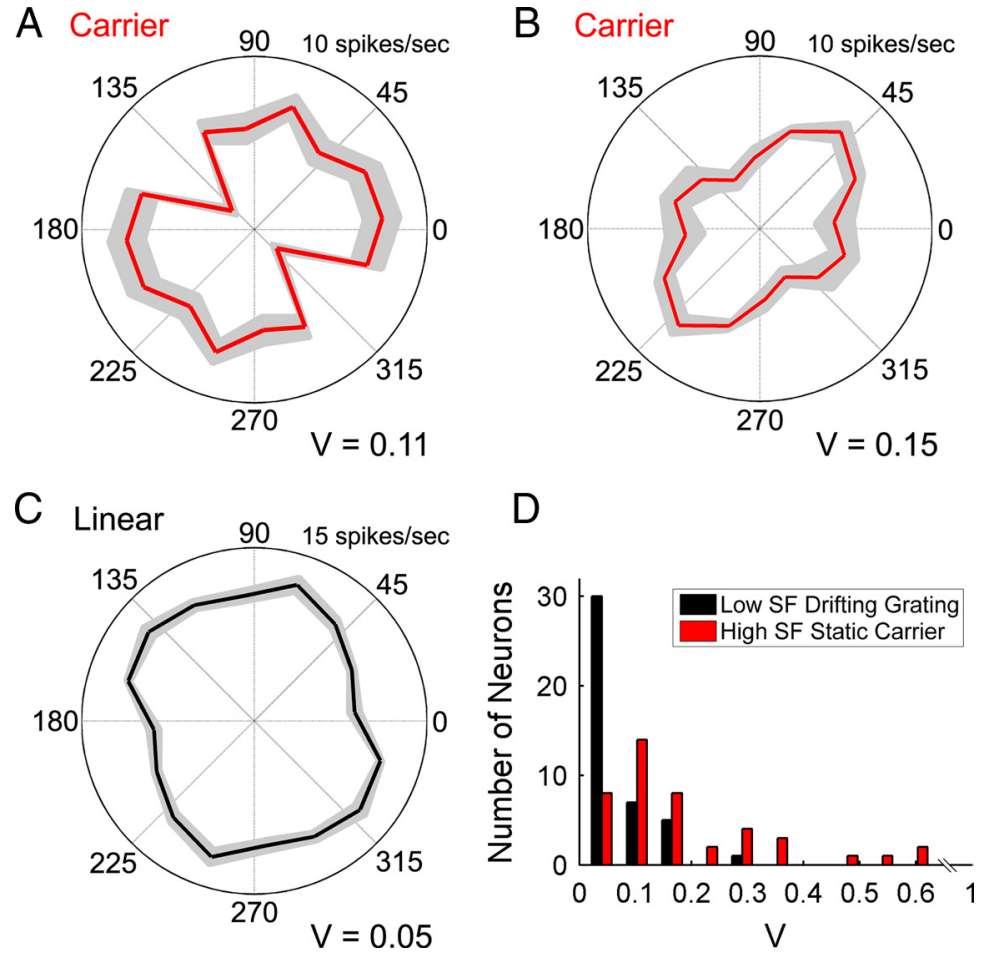

Figure 5. Orientation selectivity of Y-cells. $\boldsymbol{A}, \boldsymbol{B}$, Carrier orientation tuning curves for two $Y$-cells recorded in different experiments. Because the carrier was static in these experiments, the tuning curves are symmetric. $A$, Carrier orientation tuning for the cell shown in Figures $2 A$ and $3 A-C$. This neuron consistently responded well to all carrier orientations except for those near $135^{\circ}$. $B$, Carrier orientation tuning for a Y-cell with selectivity near the population median of $V=0.13$. C, Linear orientation tuning curve, measured using low SF drifting gratings, for the cell shown in $A$. Direction tuning was not assessed so the tuning curve is symmetric. $\boldsymbol{A}-\boldsymbol{C}$, Gray shading indicates SEM. Responses are baseline subtracted. D, Distributions of LGN Y-cell linear and carrier orientation selectivities. Larger values of $V$ indicate sharper orientation tuning.

features must be constructed from an input with at least some orientation selectivity. This has led to the suggestion that the representation of non-Fourier features is synthesized cortically from the output of primary visual cortex (von der Heydt and Peterhans, 1989; Langley et al., 1996; Mareschal and Baker, 1998b, 1999; Baker and Mareschal, 2001). However, the degree to which Y-cells are selective for carrier orientation has not been studied. To examine the orientation selectivity of LGN Y-cells, we measured linear orientation tuning curves using drifting gratings and carrier orientation tuning curves using interference patterns (Fig. 5). Linear orientation tuning was measured using drifting gratings near the cell's preferred linear SF. Carrier orientation tuning was measured with the envelope orientation and SF set near the preferred values of the linear responses and the carrier SF set near the preferred nonlinear SF measured using contrast-reversing gratings. Orientation selectivity was quantified using $\mathrm{CV}$, defining $V=1-\mathrm{CV}$ (Eq. 2) so that 0 indicates that the neuron responded equally well to all orientations and 1 indicates that it responded to only one orientation. Consistent with previous findings, the linear responses tended to be poorly selective for orientation (Fig. $5 C, D$ ), with an average $V=0.07($ median $=0.06) \pm 0.01 \mathrm{SEM}(n=43)$. The nonlinear responses, however, showed greater selectivity for carrier orientation (Fig. $5 A, B, D)$, average $V=0.19($ median $=0.13) \pm 0.03$ SEM $(n=43)$. The distributions of linear and carrier orientation selectivity were statistically different $(p<0.001$, Mann-Whitney $U$ test) (Fig. $5 D$ ). Additional analyses were conducted to show that the observed orientation preferences were not the result of astigmatism or fluctuations in firing rate unrelated to the stimulus orientation (supplemental Figs. 4, 5, available at www. jneurosci.org as supplemental material).
We also examined whether carrier orientation tuning depends on the orientation of the envelope. Carrier orientation tuning curves were measured at three envelope orientations separated by $60^{\circ}$ (envelope orientations of $5^{\circ}, 65^{\circ}$, and $125^{\circ}$ ) for $5 \mathrm{Y}$-cells and at two orthogonal envelope orientations $\left(5^{\circ}\right.$ and $\left.95^{\circ}\right)$ for 4 Y-cells. For a given Y-cell, each of the measured carrier orientation tuning curves were highly similar (supplemental Fig. $6 A, B$, available at www.jneurosci.org as supplemental material). On average, the estimates of a Y-cell's preferred carrier orientation differed by $9.9^{\circ} \pm 7.9^{\circ} \mathrm{SD}$ and the difference in orientation selectivity $(V)$ was $0.04 \pm$ 0.04 SD ( $n=19$ comparisons). The carrier orientation tuning curves were well correlated, with an average circular correlation of $0.76 \pm 0.05 \operatorname{SEM}(n=19)$. This result demonstrates that carrier orientation tuning does not depend on the envelope orientation and is consistent with previous work in cat area 18 which found the same result (Mareschal and Baker, 1998, 1999). Consistent with the independence of carrier orientation tuning and the orientation of the envelope, the preferred carrier orientation was well predicted from responses to contrast-reversing gratings (interference patterns lacking a spatial envelope) at the carrier SF (supplemental Fig. 6C,D, available at www.jneurosci.org as supplemental material). Although LGN neurons are generally thought to lack orientation selectivity, these results demonstrate that the nonlinear responses of Y-cells can show some selectivity for the orientation of the carrier of interference patterns.

\section{Comparison of carrier orientation selectivity in the LGN and cortex}

That Y-cells can show some selectivity for the orientation of the carrier of interference patterns raises the question of whether or not this degree of selectivity is comparable to what is found in cortex. To examine this, we compared carrier orientation selectivity in the LGN and area 18, a cortical area which receives feedforward input from LGN Y-cells (Stone and Dreher, 1973; Humphrey et al., 1985) and which contains a large percentage of neurons responsive to non-Fourier features (Zhou and Baker, 1994). Carrier orientation selectivity was examined in area 18 using a sample that included 32 neurons from Song and Baker (2007) and 6 we recorded for this study. For the combined sample, the average $V=0.14$ (median $=0.13$ ) $\pm 0.01 \mathrm{SEM}, n=38$. [The distributions of orientation selectivity for the two area 18 samples were not statistically different $(p=0.29$, Mann-Whitney $U$ test), average $V=0.14 \pm 0.01 \operatorname{SEM}(n=32)$ and $V=$ $0.17 \pm 0.03 \operatorname{SEM}(n=6)$, so they were analyzed together.] The sharpest carrier orientation tuning curve in the area 18 sample recorded for this study, $V=0.31$, is shown in Figure $6 C$. The distribution of carrier orientation selectivity in the LGN, described in the previous section, had an average $V=0.19$ $($ median $=0.13) \pm 0.03$ SEM $(n=43)$. Two Y-cells with tuning similar to the area 18 neuron shown in Figure $7 C$ are shown in Figure 6, $A$ and $B$. The $\mathrm{Y}$-cell with the sharpest carrier orientation 
tuning curve in the sample, $V=0.63$, is presented in supplemental Figure 3 (available at www.jneurosci.org as supplemental material). A comparison of the LGN and cortical measurements revealed that the magnitude of carrier orientation selectivity could not be used to reliably differentiate the two populations ( $p=0.46$, Mann-Whitney Utest) (Fig. 6D). It is important to note that although both LGN Y-cells and area 18 neurons show some selectivity for the orientation of the carrier of interference patterns (median $V=0.13$ ), this selectivity is substantially weaker than area 18 orientation selectivity for drifting gratings (average $V=0.73$ ) or the envelope of interference patterns (average $V=0.53$ ) (Mareschal and Baker, 1998b).

\section{TF tuning}

A previous study found that neurons in area 18 prefer higher temporal frequencies for drifting gratings than the envelopes of interference patterns (Mareschal and Baker, 1998a). However, this relationship was not found for guinea pig retinal ganglion Y-cells in vitro (Demb et al., 2001). The different cortical TF tuning curves may then bolster the argument for an intracortical origin of non-Fourier responses, as the higher grating TF cutoff may reflect properties inherent to area 18 neurons and the lower envelope TF cutoff is consistent with input from area 17 (Allison et al., 2001; Zhang et al., 2007). Recording from LGN Y-cells allowed us to examine the relationship between grating and envelope TF tuning curves in the subcortical neurons projecting to cortex. We found that LGN Y-cells, like cortical neurons, prefer higher grating than envelope temporal frequencies (Fig. 7). The preferred grating TF in the LGN was on average $9.2 \mathrm{cyc} / \mathrm{s} \pm 4.0 \mathrm{SD}(n=36)$. The preferred envelope TF was on average $4.0 \mathrm{cyc} / \mathrm{s} \pm 1.2 \mathrm{SD}(n=30)$. Y-cells in the A-layers tended to prefer higher temporal frequencies for drifting gratings (average $\mathrm{TF}_{\text {peak }}=10.3 \mathrm{cyc} / \mathrm{s} \pm 0.7 \mathrm{SD}, n=28$ ) than Y-cells in the C-layers (average $\mathrm{TF}_{\text {peak }}=5.3 \mathrm{cyc} / \mathrm{s} \pm 1.3 \mathrm{SD}, n=8$ ). The difference in preferred envelope temporal frequencies was less pronounced between the layers. The preferred envelope TF of A-layer Y-cells was $4.2 \mathrm{cyc} / \mathrm{s} \pm 1.2 \mathrm{SD}(n=24)$ and for C-layer Y-cells it was $3.2 \mathrm{cyc} / \mathrm{s} \pm 1.0 \mathrm{SD}(n=6)$. In cortex, the average preferred grating and envelope temporal frequencies were previously reported to be 6.6 and $3.8 \mathrm{cyc} / \mathrm{s}$, respectively (Mareschal and Baker, 1998a). The average preferred grating TF was higher in the LGN than cortex, consistent with previous findings (Hawken et al., 1996), but the relationship between preferred grating and envelope temporal frequencies was similar in the two areas. The median ratio of the preferred grating to envelope TF was $\sim 2.0$ for Y-cells $(n=29)$ and 1.5 for area 18 neurons $(n=30$, data extracted using DataThief from Mareschal and Baker (1998a)). The distributions of this ratio for the two areas were not statistically different ( $p=0.19$, Mann-Whitney $U$ test).

For 24 Y-cells, we measured both a contrast-reversing and an envelope TF tuning curve using the same SF for the contrastreversing gratings as the carrier of the interference patterns. Contrast-reversing gratings, which are commonly used to mea- sure the nonlinear TF tuning properties of Y-like cells (Gielen et al., 1981; Victor, 1988; Crook et al., 2008a), are two-component interference patterns that lack a spatial envelope but which have a temporal envelope that modulates at the TF of the component sinusoids. The response of Y-cells to contrast-reversing gratings and interference patterns of the form studied in this paper are qualitatively different: contrast-reversing gratings elicit responses that modulate at twice the stimulus TF (supplemental Fig. 1, available at www.jneurosci.org as supplemental material) whereas interference patterns elicit responses that modulate at the envelope TF (Fig. 3) (Demb et al., 2001). Despite that qualitatively different response profiles are elicited by contrast-reversing gratings and interference patterns, if Y-cells are tuned for modulation (envelope) frequency then TF tuning curves measured with these different stimuli should be the same. Consistent with this expectation, the contrast-reversing and envelope TF tuning curves of Y-cells were highly similar. The average ratio of the preferred envelope to contrast-reversing TF was $1.35 \pm 0.33 \mathrm{SD}$, and the median variance $\left(r^{2}\right)$ in one of these tuning curves explained by the other was $73 \%$ (Fig. $7 A, C$ ).

\section{Discussion}

In this study we examined the responses of cat LGN X- and Y-cells to Fourier and non-Fourier image features. Whereas X-cells only responded to Fourier features, Y-cells responded to both Fourier and non-Fourier features. Y-cell linear and nonlinear spatiotemporal tuning properties were then characterized and shown to be similar to those of cortical area 18 neurons responsive to interference patterns. Although area 18 responses to interference patterns have previously been attributed to intracortical processing of the output of area 17, the present findings suggest that LGN Y-cells, the principle LGN input to area 18, may provide the nonlinear signal from which cor- 
A

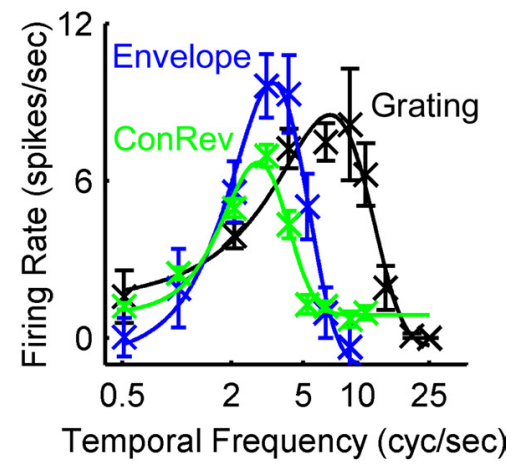

B

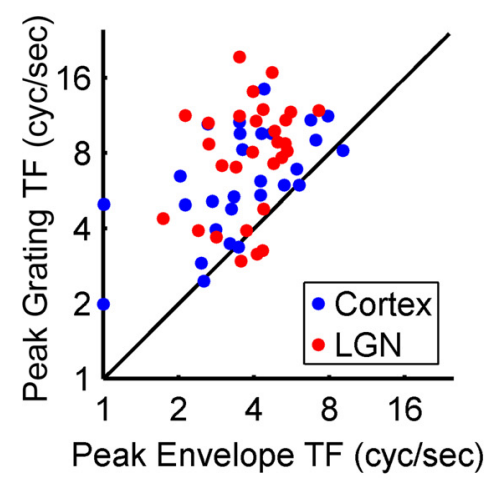

C

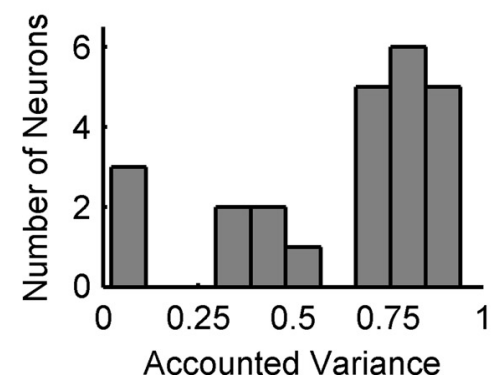

Figure 7. Temporal frequency tuning.A, Three TF tuning curves for a single LGNY-cell. Black curve shows drifting grating responses, blue curve envelope responses, green curve contrast-reversing responses at the carrier SF used for the interference patterns. Responses are baseline subtracted, and error bars show SEM. Note that the grating tuning curve peaks and cuts off at a higher TF than the envelope tuning curve, as well as the similarity between the envelope and contrast-reversing tuning curves $\left(r^{2}=0.74\right)$. B, Scatter plot of preferred grating versus envelope temporal frequencies in the LGN (red dots) and cortex (area 18; blue dots). Note that the preferred grating TF is consistently higher than the preferred envelope TF in both areas, and that the distributions overlap substantially. Cortical data are replotted from Mareschal and Baker (1998a). C, Histogram of accounted variance between the envelope and contrast-reversing TF tuning curves for $24 \mathrm{Y}$-cells, median $r^{2}=0.73$.

tical responses to non-Fourier features are generated. These results thus support the hypothesis that representations of Fourier and nonFourier features emerge in parallel through distinct cellular pathways originating at the retina. Just as the cortical representation of Fourier features is thought to be synthesized from the output of LGN $\mathrm{X}$-cells, the cortical representation of non-Fourier features may be synthesized directly from the output of LGN Y-cells.

\section{Subcortical and cortical responses to interference patterns are similar}

A previous study found that retinal ganglion Y-cells respond to non-Fourier motion (Demb et al., 2001), but the suggestion that Y-cells may underlie the representation of non-Fourier features has not been widely accepted. This is because cortical electrophysiology in both cats (Mareschal and Baker, 1998b) and monkeys (von der Heydt and Peterhans, 1989) as well as human psychophysics (Langley et al., 1996; Dakin and Mareschal, 2000) have suggested that the representation of non-Fourier features is constructed from the output of neurons with cortex-like tuning properties (Baker and Mareschal, 2001). The present findings, which demonstrate that the non-Fourier responses of LGN Y-cells are similar to those observed in cortex, may resolve this discrepancy.

We first demonstrated that the SF preferences of both the linear and nonlinear responses of LGN Y-cells and area 18 neurons are similar. The sensitivity of LGN neurons to interference patterns was then quantified using the Gini coefficient from ROC analysis. All of the recorded Y-cells but none of the X-cells could reliably signal the presentation of an interference pattern, quantifying an earlier report that Y-cells respond to interference patterns (Demb et al., 2001) and importantly demonstrating that $\mathrm{X}$-cells do not possess an overlooked nonlinearity that would make them sensitive to non-Fourier features. Y-cell tuning properties were then characterized and compared with those of cortical neurons from area 18 . Both populations were found to possess a similar degree of selectivity for the orientation of the high SF carrier of interference patterns. Carrier orientation selectivity has been one of the principle findings supporting the hypothesis that area 18 neurons synthesize their non-Fourier responses from the output of area 17, but this result shows that comparable orientation selectivity is found subcortically. Given the retinal origin of the Y-cell pathway, one possibility is that biases in carrier orientation tuning originate with the bipolar cells that synapse onto retinal ganglion $\alpha(\mathrm{Y})$-cells. Elongated bipolar cell receptive fields would give rise to a bias in carrier orientation tuning which could pass forward to LGN Y-cells and, in turn, to cortical neurons. Consistent with this possibility, a previous study examining the orientation selectivity of the nonlinear responses of cat retinal ganglion Y-cells with high SF contrast-reversing gratings (Thibos and Levick, 1985) reported orientation biases similar to what we found in the LGN and others have found in cortex for the carrier of interference patterns (Mareschal and Baker, 1998b; Song and Baker, 2007). A feedforward origin to this orientation bias is also consistent with our finding that carrier orientation preference is well predicted from responses to contrast-reversing gratings. Finally, we showed that LGN Y-cells have the same systematic difference in their grating and envelope TF preferences as area 18 neurons (Mareschal and Baker, 1998a). We were able to predict this difference from responses to high SF contrast-reversing gratings. Although this relationship was not observed for in vitro guinea pig retinal ganglion Y-cells (Demb et al., 2001), other studies that presented drifting and contrast-reversing gratings have found TF results similar to our own in primate retinal ganglion (Crook et al., 2008a) and LGN Y-like cells (Gielen et al., 1981). Together with the known cortical projection patterns of LGN Y-cells, these results suggest that the LGN input to area 18 carries a nonlinear signal appropriate for generating cortical responses to non-Fourier image features.

\section{Cortical processing of the $\mathrm{X}$ - and $\mathrm{Y}$-cell pathways}

Area 17 of the cat receives a mixture of X-and Y-cell input but its responses seem to be dominated by its linear X-cell input, whereas area 18 receives predominantly $\mathrm{Y}$-cell input and displays characteristic Y-like nonlinearities (Stone and Dreher, 1973; Humphrey et al., 1985; Ferster, 1990; Ferster and Jagadeesh, 1991). At the population level these areas represent qualitatively 
different image features; area 17 represents Fourier features and area 18 represents both Fourier and non-Fourier features (Zhan and Baker, 2006; Issa et al., 2008). Despite this difference, a common principle appears to govern the cortical transformation of the LGN signal by individual neurons in areas 17 and 18: the alignment of LGN receptive fields along oriented axes in space and time (Hubel and Wiesel, 1962; Tretter et al., 1975). As such, the difference in the image features represented by these areas may emerge because of the qualitative difference in their LGN input. Specifically, area 17 may represent Fourier features because its responses are dominated by X-cell input and area 18 may represent both Fourier and non-Fourier features because its responses are dominated by Y-cell input. From this perspective, cat areas 17 and 18 may be considered complementary primary visual areas, acting in parallel to provide functionally distinct representations of the visual scene (Tretter et al., 1975; Ferster and Jagadeesh, 1991).

\section{Relevance to the primate visual system}

There has been considerable controversy over whether or not there is a primate homolog to the Y-cell, and so this may raise doubts about the role of Y-cells in generating sensitivity to nonFourier features in the primate. However, recent studies have demonstrated the existence of at least two distinct types of Y-like primate retinal ganglion cells, one of which is the parasol cell (Petrusca et al., 2007; Crook et al., 2008a,b). These cells display the characteristic physiological properties of Y-cells, including frequency-doubled responses to high SF contrast-reversing gratings. Consistent with the finding that parasol cells but not midget cells display frequency-doubled responses (Petrusca et al., 2007; Crook et al., 2008a), Y-like frequency-doubled responses have been found principally in the magnocellular layers of the primate LGN (Kaplan and Shapley, 1982; Levitt et al., 2001). The $\sim 10$ fold difference between the preferred linear and nonlinear spatial frequencies we found in the cat LGN and others have reported in cat area 18 (Mareschal and Baker, 1999) also appears consistent with the responses of both primate parasol cells (Crook et al., 2008a) and LGN Y-cells (Gielen et al., 1981). Y-like cells may thus underlie sensitivity to non-Fourier features in the primate visual system (Petrusca et al., 2007; Rosenberg and Talebi, 2009).

\section{References}

Adelson EH, Bergen JR (1985) Spatiotemporal energy models for the perception of motion. J Opt Soc Am A 2:284-299.

Albright TD (1992) Form-cue invariant motion processing in primate visual cortex. Science 255:1141-1143.

Allison JD, Smith KR, Bonds AB (2001) Temporal-frequency tuning of cross-orientation suppression in the cat striate cortex. Vis Neurosci 18:941-948.

Baker CL Jr, Mareschal I (2001) Processing of second-order stimuli in the visual cortex. Prog Brain Res 134:171-191.

Baker TI, Issa NP (2005) Cortical maps of separable tuning properties predict population responses to complex visual stimuli. J Neurophysiol 94:775-787.

Bellefeuille A, Faubert J (1998) Independence of contour and biologicalmotion cues for motion-defined animal shapes. Perception 27:225-235.

Brainard DH (1997) The psychophysics toolbox. Spat Vis 10:433-436.

Chubb C, Sperling G (1988) Drift-balanced random stimuli: a general basis for studying non-Fourier motion perception. J Opt Soc Am A 5:1986-2007.

Crook JD, Peterson BB, Packer OS, Robinson FR, Troy JB, Dacey DM (2008a) Y-cell receptive field and collicular projection of parasol ganglion cells in macaque monkey retina. J Neurosci 28:11277-11291.

Crook JD, Peterson BB, Packer OS, Robinson FR, Gamlin PD, Troy JB, Dacey DM (2008b) The smooth monostratified ganglion cell: evidence for spatial diversity in the Y-cell pathway to the lateral geniculate nucleus and superior colliculus in the macaque monkey. J Neurosci 28:12654-12671.
Dakin SC, Mareschal I (2000) Sensitivity to contrast modulation depends on carrier spatial frequency and orientation. Vision Res 40:311-329.

Demb JB, Zaghloul K, Sterling P (2001) Cellular basis for the response to second-order motion cues in Y retinal ganglion cells. Neuron 32:711-721.

Fawcett T (2006) An introduction to ROC analysis. Pattern Recognit Lett 27:861-874.

Ferster D (1990) X-and Y-mediated synaptic potentials in neurons of areas 17 and 18 of cat visual cortex. Vis Neurosci 4:115-133.

Ferster D, Jagadeesh B (1991) Nonlinearity of spatial summation in simple cells of areas 17 and 18 of cat visual cortex. J Neurophysiol 66:1667-1679.

Fleet DJ, Langley K (1994) Computational analysis of non-Fourier motion. Vision Res 34:3057-3079.

Gielen CC, van Gisbergen JA, Vendrik AJ (1981) Characterization of spatial and temporal properties of monkey LGN Y-cells. Biol Cybern 40:157-170.

Hawken MJ, Shapley RM, Grosof DH (1996) Temporal-frequency selectivity in monkey visual cortex. Vis Neurosci 13:477-492.

Hegdé J, Albright TD, Stoner GR (2004) Second-order motion conveys depth-order information. J Vis 4:838-842.

Hochstein S, Shapley RM (1976a) Quantitative analysis of retinal ganglion cell classifications. J Physiol 262:237-264.

Hochstein S, Shapley RM (1976b) Linear and nonlinear spatial subunits in Y cat retinal ganglion cells. J Physiol 262:265-284.

Hubel DH, Wiesel TN (1962) Receptive fields, binocular interaction and functional architecture in the cat's visual cortex. J Physiol 160:106-154.

Humphrey AL, Sur M, Uhlrich DJ, Sherman SM (1985) Termination patterns of individual $\mathrm{X}$ - and $\mathrm{Y}$-cell axons in the visual cortex of the cat: projections to area 18 , to the $17 / 18$ border region, and to both areas 17 and 18. J Comp Neurol 233:190-212.

Issa NP, Rosenberg A, Husson TR (2008) Models and measurements of functional maps in V1. J Neurophysiol 99:2745-2754.

Kaplan E, Shapley RM (1982) X and Y cells in the lateral geniculate nucleus of macaque monkeys. J Physiol 330:125-143.

Langley K, Fleet DJ, Hibbard PB (1996) Linear filtering precedes nonlinear processing in early vision. Curr Biol 6:891-896.

Langley K, Fleet DJ, Hibbard PB (1998) Linear and nonlinear transparencies in binocular vision. Proc Biol Sci 265:1837-1845.

Leventhal AG, Wang Y, Schmolesky MT, Zhou Y (1998) Neural correlates of boundary perception. Vis Neurosci 15:1107-1118.

Levitt JB, Schumer RA, Sherman SM, Spear PD, Movshon JA (2001) Visual response properties of neurons in the LGN of normally reared and visually deprived macaque monkeys. J Neurophysiol 85:2111-2129.

Lu ZL, Sperling G (2001) Three-systems theory of human visual motion perception: review and update. J Opt Soc Am A Opt Image Sci Vis 18:2331-2370.

Mante V, Carandini M (2005) Mapping of stimulus energy in primary visual cortex. J Neurophysiol 94:788-798.

Mareschal I, Baker CL Jr (1998a) Temporal and spatial response to secondorder stimuli in cat area 18. J Neurophysiol 80:2811-2823.

Mareschal I, Baker CL Jr (1998b) A cortical locus for the processing of contrast-defined contours. Nat Neurosci 1:150-154.

Mareschal I, Baker CL Jr (1999) Cortical processing of second-order motion. Vis Neurosci 16:527-540.

Orger MB, Smear MC, Anstis SM, Baier H (2000) Perception of Fourier and non-Fourier motion by larval zebrafish. Nat Neurosci 3:1128-1133.

Petrusca D, Grivich MI, Sher A, Field GD, Gauthier JL, Greschner M, Shlens J, Chichilnisky EJ, Litke AM (2007) Identification and characterization of a Y-like primate retinal ganglion cell type. J Neurosci 27:11019-11027.

Price DJ, Ferrer JM, Blakemore C, Kato N (1994) Functional organization of corticocortical projections from area 17 to area 18 in the cat's visual cortex. J Neurosci 14:2732-2746.

Rivest J, Cavanagh P (1996) Localizing contours defined by more than one attribute. Vision Res 36:53-66.

Rosenberg A, Talebi V (2009) The primate retina contains distinct types of Y-like ganglion cells. J Neurosci 29:5048-5050.

Sáry G, Vogels R, Orban GA (1993) Cue-invariant shape selectivity of macaque inferior temporal neurons. Science 260:995-997.

Song Y, Baker CL Jr (2007) Neuronal response to texture- and contrastdefined boundaries in early visual cortex. Vis Neurosci 24:65-77.

Stone J, Dreher B (1973) Projection of X- and Y-cells of the cat's lateral geniculate nucleus to areas 17 and 18 of visual cortex. J Neurophysiol 36:551-567. 
Sur M, Sherman SM (1982) Linear and nonlinear W-cells in C-laminae of the cat's lateral geniculate nucleus. J Neurophysiol 47:869-884.

Tanaka H, Ohzawa I (2006) Neural basis for stereopsis from second-order contrast cues. J Neurosci 26:4370-4382.

Theobald JC, Duistermars BJ, Ringach DL, Frye MA (2008) Flies see second-order motion. Curr Biol 18:R464-R465.

Thibos LN, Levick WR (1985) Orientation bias of brisk-transient y-cells of the cat retina for drifting and alternating gratings. Exp Brain Res 58:1-10.

Tretter F, Cynader M, Singer W (1975) Cat parastriate cortex: a primary or secondary visual area. J Neurophysiol 38:1099-1113.

Victor JD (1988) The dynamics of the cat retinal Y cell subunit. J Physiol 405:289-320 von der Heydt R, Peterhans E (1989) Mechanisms of contour perception in monkey visual cortex. I. Lines of pattern discontinuity. J Neurosci 9:1731-1748

Watson AB, Ahumada AJ Jr (1983) A look at motion in the frequency domain. NASA Technical Memorandum TM-84352.

Zhan CA, Baker CL Jr (2006) Boundary cue invariance in cortical orientation maps. Cereb Cortex 16:896-906.

Zhang JX, Rosenberg A, Mallik AK, Husson TR, Issa NP (2007) The representation of complex images in spatial frequency domains of primary visual cortex. J Neurosci 27:9310-9318.

Zhou YX, Baker CL Jr (1994) Envelope-responsive neurons in areas 17 and 18 of cat. J Neurophysiol 72:2134-2150. 